Complementary enantioselectivity profiles of chiral cinchonan carbamate selectors with distinct carbamate residues and their implementation in enantioselective two-dimensional high-performance liquid chromatography of amino acids

\title{
Woiwode, Ulrich
}

2018-07-13

Woiwode , U , Ferri , M , Maier , N M , Lindner , W \& Lämmerhofer , M 2018 , ' Complementary enantioselectivity profiles of chiral cinchonan carbamate selectors with distinct carbamate residues and their implementation in enantioselective two-dimensional high-performance liquid chromatography of amino acids ' , Journal of Chromatography. A , vol. 1558 , pp. 29-36 . https://doi.org/10.1016/j.chroma.2018.04.061

http://hdl.handle.net/10138/314406

https://doi.org/10.1016/j.chroma.2018.04.061

cc_by_nc_nd

acceptedVersion

Downloaded from Helda, University of Helsinki institutional repository.

This is an electronic reprint of the original article.

This reprint may differ from the original in pagination and typographic detail.

Please cite the original version. 


\section{Accepted Manuscript}

Title: Complementary enantioselectivity profiles of chiral cinchonan carbamate selectors with distinct carbamate residues and their implementation in enantioselective two-dimensional high-performance liquid chromatography of amino acids

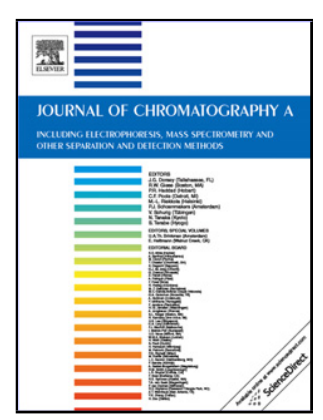

Authors: Ulrich Woiwode, Martina Ferri, Norbert M. Maier, Wolfgang Lindner, Michael Lämmerhofer

PII: $\quad$ S0021-9673(18)30522-3

DOI: $\quad$ https://doi.org/10.1016/j.chroma.2018.04.061

Reference: $\quad$ CHROMA 359360

To appear in: $\quad$ Journal of Chromatography A

Received date: $\quad 15-3-2018$

Revised date: 23-4-2018

Accepted date: $\quad$ 26-4-2018

Please cite this article as: Ulrich Woiwode, Martina Ferri, Norbert M.Maier, Wolfgang Lindner, Michael Lämmerhofer, Complementary enantioselectivity profiles of chiral cinchonan carbamate selectors with distinct carbamate residues and their implementation in enantioselective two-dimensional highperformance liquid chromatography of amino acids, Journal of Chromatography A https://doi.org/10.1016/j.chroma.2018.04.061

This is a PDF file of an unedited manuscript that has been accepted for publication. As a service to our customers we are providing this early version of the manuscript. The manuscript will undergo copyediting, typesetting, and review of the resulting proof before it is published in its final form. Please note that during the production process errors may be discovered which could affect the content, and all legal disclaimers that apply to the journal pertain. 
Complementary enantioselectivity profiles of chiral cinchonan carbamate selectors with distinct carbamate residues and their implementation in enantioselective twodimensional high-performance liquid chromatography of amino acids

Ulrich Woiwode ${ }^{a}$, Martina Ferria,b, Norbert M. Maier ${ }^{c}$, Wolfgang Lindner ${ }^{d, e}$, Michael Lämmerhofer ${ }^{\mathrm{a}}$

a Institute of Pharmaceutical Sciences, Pharmaceutical (Bio-)Analysis, University of Tübingen, Auf der Morgenstelle 8, 72076 Tübingen, Germany

b Department of Pharmaceutical Sciences, University of Perugia, Via Fabretti 48, 06123Perugia, Italy

${ }^{c}$ Department of Chemistry, University of Helsinki, PO. Box 55, FI-00014, Finland

${ }^{d}$ Lindner Consulting GmbH, Ziegelofengasse 37, 3400 Klosterneuburg, Austria

e Institute of Analytical Chemistry, University of Vienna, Waehringerstrasse 38, 1090 Vienna, Austria

${ }^{*}$ Author for correspondence:

Prof. Dr. Michael Lämmerhofer

Institute of Pharmaceutical Sciences, Pharmaceutical (Bio-)Analysis

University of Tübingen

Auf der Morgenstelle 8

72076 Tübingen, Germany

T +4970712978793, F +497071294565

e-mail: michael.laemmerhofer@uni-tuebingen.de 


\section{Highlights}

1. Seven cinchonan derived chiral stationary phases (CSPs) tested

2. Complementarity of CSPs evaluated by principal component analysis

3. Two CSPs with most orthogonal enantioselectivities selected for 2D-HPLC

4. Quinine tert-butyl and 2,6-diisopropylphenyl carbamates for comprehensive 2D-HPLC

5 . Orthogonality increased with opposite configurations of selectors in ${ }^{1} D$ and ${ }^{2} D$

\section{Abstract}

A cardinal requirement for effective 2D-HPLC separations is sufficient complementarity in the retention profiles of first and second dimension separations. It is shown that retention and enantioselectivity of chiral selectors derived from cinchona alkaloids can be conveniently modulated by structural variation of the carbamate residue of the quinine/quinidine carbamate ligand of such chiral stationary phases (CSP). A variety of aliphatic and aromatic residues have been tested in comparison to non-carbamoylated quinine CSP. Various measures of orthogonality have been utilized to derive the CSP that is most complementary to the tert-butylcarbamoylated quinine CSP (tBuCQN CSP), which is commercially available as Chiralpak QN-AX column. It turned out that O-9-(2,6-diisopropylphenylcarbamoyl)modified quinine is most promising in this respect. Its implementation as a complementary CSP for the separation of amino acids derivatized with Sanger's reagent $(2,4-$ dinitrophenylated amino acids) in the first dimension combined with a tBuCQN CSP in the second dimension revealed successful enantiomer separations in a comprehensive chiral $\times$ chiral 2D-HPLC setup. However, the degree of complementarity could be greatly enhanced when simultaneously the absolute configurations were exchanged from quinine to quinidine in the chiral selector of the first dimension separation resulting in opposite elution orders of the enantiomers in the two dimensions. The advantage of such a chiral $\times$ chiral over achiral×chiral 2D-HPLC setup, amongst others, is the perfect compatibility of the mobile phase because in both dimensions the identical eluent can be used.

\section{Keywords}


Multidimensional HPLC separations, chiral stationary phase, quinine, quinidine, amino acids, two-dimensional chromatography 


\section{Introduction}

HPLC enantiomer separation has reached high levels of maturation. A large variety of excellent chiral stationary phases (CSPs) are nowadays available. They can very well cope with the demand of efficient enantiomer separation of distinct chiral compounds relevant to drug discovery, food industry (sweeteners, flavors), agriculture (pesticides, herbicides), clinical analysis (metabolites) and other fields [1]. Unfortunately, the unique enantioselective column, which exhibits enantioselectivity for all chiral analytes and can separate any chiral compound, does not exist. Hence, to successfully deal with the huge structural variety of chiral analytes, several distinct CSPs and columns, respectively, are required, because even minor variations in the analyte structure may lead to loss of enantioselectivity. Alternatives are then indispensable.

Under this background it is not surprising that numerous CSPs and "chiral columns" are nowadays commercially available [2]. Thereby, it is quite common to take a molecule from the natural chiral pool as a chiral selector and decorate it with distinct structural features which are favorable for enantiorecognition. Complementary selectors are thus obtained which expand the spectrum of application. For example, this concept has been utilized for polysaccharide based CSPs. Cellulose and amylose are derivatized to ester and carbamates, respectively, and to further expand the application spectrum different aryl residues (dimethylphenyl, chloro/methyl-phenyl, dichlorophenyl) are attached as pendant groups [3-5]. CSPs based on macrocyclic antibiotics incorporate immobilized versions of vancomycin, teicoplanin, teicoplanin aglycone and ristocetin as chiral selectors [6, 7]. Numerous derivatives of cyclodextrins have been proposed as CSPs [8,9], and crown ether CSPs are also commercialized in different structural variants [10, 11]. Similarly, cinchona alkaloids can be derivatized with distinct functionalities to introduce interaction sites that make them complementary in their application profiles [12-15].

Aside from distinct structural decorations of selector moieties, application profiles can also be expanded by combination of different chiral columns. One simple option represents the inline 
coupling of columns (achiral and chiral, chiral and chiral) [16-18]. More advanced is the column coupling in 2D-HPLC to resolve components not resolved in a single chromatographic dimension by a chiral column. Heart-cut [19-21], multiple heart-cut [22-30] high-resolution sampling (selective comprehensive), offline [31] and online comprehensive [32] are the various modalities which can be implemented to achieve the goal of full separation of difficult sample mixtures.

Multidimensional separations require orthogonal retention and separation principles in order to accomplish the full power of resolution and evenly spread analyte spots over the 2dimensional separation space [33]. In this report, we elucidate the possibility of generation of complementary retention and selectivity profiles of quinine and quinidine carbamate based weak anion-exchange type CSPs via structural variation of carbamate residues (Fig. 1). The goal is to identify a CSP which can be beneficially combined in 2D-HPLC with the commercially available tert-butylcarbamoyl quinine CSP. Such a combination of wellmatched CSPs may then allow single-run 2D-LC resolution of enantiomeric mixtures which fail to be resolved on a single column. As an added benefit, such a CSP combination can be operated with the same mobile phase. This would ensure optimal compatibility between the two dimensions, thus avoiding mobile phase-related issues upon column-to-column transfer in 2D-LC. A generic system for column screening with extended coverage should be the result of such endeavor.

\section{Materials and methods}

\subsection{Materials}

The tert-butylcarbamoyl quinine-based CSP $(5 \mu \mathrm{m}, 100 \AA)$ (Fig. 1) was a prototype of the commercially available Chiralpak QN-AX (from Chiral Technologies Europe, Illkirch, France). The synthesis of the other CSPs (5 $\mu \mathrm{m}, 100 \AA$ ) (Fig. 1) followed protocols described earlier [14, 34, 35]. 
Methanol and acetonitrile in HPLC grade quality were form VWR (Fontenay-sous-Bois, France). 2,2'-Azobis-(2-methylpropionitrile) (AIBN), ammonium acetate and acetic acid were from Sigma (Munich, Germany). Highly purified water was supplied by an Elga PureLab Ultra purification system (Celle, Germany).

Sodium hydrogen carbonate, sodium carbonate, and 1-fluoro-2,4-dinitrobenzene (DNFB) were also from Sigma. Racemic and (S)-amino acids were obtained from Sigma, Roth (Karlsruhe, Germany) and Merck (Darmstadt, Germany). Dichloromethane (HPLC-grade) was from Fisher Scientific (Loughborough, UK).

\subsection{Derivatization of amino acids with Sanger's reagent}

Racemic and $(S)$-configurated amino acids $(0.5 \mathrm{mg} / \mathrm{mL})$ were dissolved separately in $0.1 \mathrm{M}$ sodium carbonate buffer $\mathrm{pH} 9.4$ and $500 \mu \mathrm{L}$ of each solution were mixed with $125 \mu \mathrm{L} 1$ fluoro-2,4-dinitrobenzene (DNFB) $(2.5 \% \mathrm{v} / \mathrm{v}$ in acetonitrile). After $2 \mathrm{~h}$ of reaction at room temperature, $350 \mu \mathrm{L}$ of reaction mixture were added to $350 \mu \mathrm{L}$ of dichloromethane to quench the reaction by removal of DNFB into the organic layer. Before retrieving the aqueous layer, $17.5 \mu \mathrm{L}$ concentrated ammonia was added to retain labeled amino acids. Samples were then mixed as required and diluted 1:10 (v/v) with mobile phase. Standards for peak identification and confirmation of elution order were prepared by spiking the $(S)$-enantiomer to the respective racemic amino acid in this dilution step.

\subsection{D-LC instrument}

Online 2D-LC was done with an Agilent 1290 Infinity II 2D-LC Solution from Agilent Technologies (Waldbronn, Germany) with following components: ${ }^{1} \mathrm{D}-$ pump, quaternary lowpressure gradient UHPLC pump (Flexible pump, G7104A), ${ }^{2} \mathrm{D}$-pump, binary high-pressure gradient UHPLC pump (High Speed Pump, G7120A); autosampler (Multisampler, G7167B); 
${ }^{1} \mathrm{D}$ - and ${ }^{2} \mathrm{D}$-column compartment (Multicolumn Thermostat, G7116B); ${ }^{1} \mathrm{D}$-detector, UV (Variable wavelength detector, G7114B) with $14 \mu \mathrm{L}$ flow cell (G1314-60186); ${ }^{2} \mathrm{D}$-detector, DAD (G7117B) with $1 \mu \mathrm{L}$ flow cell (G4212-60008). First and second dimension were interfaced with a dual two-position four port valve with two $60 \mu \mathrm{L}$ loops (items in G4236A). A pressure release kit (G4236-60010) was installed between UV-detector and interface. Open Lab CDS Rev. C.01.07SR3 and LC-Image Version 2.6b3 LCxLC-HRMS from GC Image (Lincoln, NE, USA) were used for processing.

\subsection{D-LC method}

Chiral stationary phases with enhanced stability [36] were used for separations at $55^{\circ} \mathrm{C}$ in both dimensions. For first separation, either a DIPPCQD CSP $\left(0.26 \mathrm{mmol} / \mathrm{g}, 0.88 \mu \mathrm{mol} / \mathrm{m}^{2}\right)$ or a DIPPCQN CSP $\left(0.25 \mathrm{mmol} / \mathrm{g}, 0.82 \mu \mathrm{mol} / \mathrm{m}^{2}\right)$ immobilized to fully porous silica $(5 \mu \mathrm{m}$, $100 \AA$ pore size, $150 \times 4 \mathrm{~mm}$ ID column) was used. Fast separations in the second dimension were done with a tBuCQN CSP $\left(0.19 \mathrm{mmol} / \mathrm{g}, 1.0 \mu \mathrm{mol} / \mathrm{m}^{2}\right)$ with wide pore silica (fully porous particles, $5 \mu \mathrm{m}, 200 \AA$ pore size in a $33 \times 4.6 \mathrm{~mm}$ ID column. LC-parameters were as follows: ${ }^{1} \mathrm{D} /{ }^{2} \mathrm{D}$-mobile phase, methanol/acetic acid/ammonium acetate (98/2/0.5, $\mathrm{v} / \mathrm{v} / \mathrm{w}) ;{ }^{1} \mathrm{D}$-flow rate, $0.1 \mathrm{~mL} / \mathrm{min}$; ${ }^{2} \mathrm{D}$-flow rate, $2 \mathrm{~mL} / \mathrm{min}$; injection volume, $20 \mu \mathrm{L}$ of DNPamino acid mixture; ${ }^{1} \mathrm{D} /{ }^{2} \mathrm{D}$-column temperature, $55^{\circ} \mathrm{C}$; modulation, $60 \mu \mathrm{L}$ fractions of ${ }^{1} \mathrm{D}$ effluent every 60 seconds; ${ }^{1} D$-detection, UV at $360 \mathrm{~nm}$; ${ }^{2}$ D-detection, DAD at $360 \mathrm{~nm}$.

\subsection{Statistics}

Multivariate statistics was performed with SIMCA-P+ (version 12) (Umetrics, Umea, Sweden). About 50 test compounds were analyzed on the various CSPs (see Suppl. Material, Table S1). Before statistics the data were scaled by unit variance. Principal component analysis (PCA-X) was carried out on data matrices of retention factors $k_{1}$ and separation factors $\alpha$ as variables $(K)$ for the seven CSPs tested as observations $(N=7)$. The 
number of cross-validation groups was 7 . The confidence level on the parameters was $95 \%$ and the significance level for DModX and Hotelling's T2 was $P=0.05$. Coefficients were scaled and centered. The distance to model is normalized in units of standard deviation. Compounds with a poor model fit $\left(Q^{2} V X<0.5\right)$ have been excluded from the analysis for the final model.

Pearson correlation coefficients of the retention factors on the distinct CSPs (derived to elucidate the orthogonality of different CSPS) were calculated using SPSS version 23 (IBM). Wilcoxon signed-rank test for paired sample comparisons was performed with OriginPro (Origin Lab Corporation, Northampton, MA, USA).

\section{Results and discussion}

\subsection{Complementarity of chiral selectors with distinct carbamate residues}

\subsubsection{Complementarity of enantioselectivity}

In order to generate a generic 2D-HPLC setup with an extended scope of chiral separation for acidic chiral analytes, two complementary CSPs should be combined in a comprehensive chiral $\times$ chiral 2D-HPLC approach. Along this line, the focus of this study was to identify a CSP which shows in terms of enantioselectivity and application spectrum the highest level of complementarity to that of the tBuCQN CSP. This implies that the CSP to be combined with the benchmark tBuCQN CSP should be able to separate chiral acids which are typically not or only poorly resolved by the tBuCQN CSP alone. Partly such problems have previously been addressed by inline coupling of columns [37]. Herein, two columns should be combined to realize a 2D-LC separation system capable of resolving the enantiomers of chiral acids of a wide structural variability.

Quinine and a number of differently decorated quinine carbamates were examined as chiral selectors to achieve this goal. The corresponding CSP structures and abbreviations used herein can be found in Fig. 1. Some of the carbamate residues introduce steric bulk (tBu, cHex, 1Adam), others provide additional $\pi$ - $\pi$-interaction sites for aromatic counterparts of 
analytes, or provide a combination of these interactions (DIPP, Trit). The presence of these distinct structural elements in the latter CSPs increases their chance of being orthogonal in terms of enantioselectivity. Consequently, the probability that compounds which are not separated by tBuCQN CSP can be resolved on such alternative CSPs is rising.

To document the complementarity of the CSPs depicted in Fig. 1, separation factors (Supplementary Table S1) were subjected to PCA-X and the results are given in Fig. 2 and Supplementary Table S2. It can be seen in Suppl. Table S2 that the quality of fit and predictive power of the model increases with the number of components. Three components gave the best model, while with more components the predictive power dropped again. The cumulative $Q^{2}$ of the cross-validation process reached a value of 0.682 which is acceptable for the current purpose to show complementarity and grouping of CSPs. $48.8 \%$ of the variance in the test set can be explained by the $1^{\text {st }}$ component, 32.7 by the $2^{\text {nd }}$ and $11 \%$ by the third. A bi-Plot (a superposition of score plot, green symbols, and loadings plot, black symbols) is shown in Fig. 2. From the scores it can be seen that the CSPs which are clustered together in the bi-plot are more similar in terms of separation factors. In contrast, those being farther away from each other are more dissimilar; thus they have higher orthogonality in their enantiomer separation capability. A combination of two columns being farther away from each in the bi-plot seems to be advantageous for a 2D-HPLC setup with broad applicability scope. Essentially we see 3 clusters: i) tBuCQN, cHexCQN and 1 AdamCQN are more closely together in the score plot, thus more similar; ii) DIPPCQN and TritCQN have similar scores and should be similar in enantiorecognition power, but significantly different compared to the CSP with steric aliphatic residue (i.e. tBuCQN and others), and iii) QN- and N-Me-tBuCQN are located close to each other in the score plot as well. The loadings explain the positions of the observations in the score plot and provide information on which analytes are responsible for the particular grouping of CSPs in the biplot. The loadings correlate the variables ( $k$ of analytes) with the observations (scores). Inspection of the bi-plot in Fig. 2 thus readily allows to pinpoint the analytes responsible for the clustering of these three CSP groups. For example, DNB-Pro has a strong negative 
influence on PC1 and is strongly correlated with QN CSP and N-Me-tBuCQN CSP. These two CSPs show the highest separation factor for DNB-Pro. DNB- and Fmoc-amino acids strongly load on PC1 having a strong positive influence. They are correlated with CSPS having an aliphatic carbamate residue (tBu, cHex, 1Adam). Compounds such as profens, aryloxy carboxylic acids and $\mathrm{N}$-arylamino acids show a strong positive influence on PC2 and are clustering together DIPPCQN and TritCQN CSPs.

For 2D-HPLC a CSP of a different cluster than tBuCQN should be combined with it, giving presumably the most generic enantioselective separation system. PCA suggests four CSPs to be selected for this purpose (QN, N-Me-tBuCQN, DIPPCQN or TritCQN). To find the best decision, a closer look into the separation factors was undertaken. Chiral compounds well separated by tBuCQN CSP do not need to be further considered in this evaluation. However, the second CSP should be able to resolve chiral compounds not separated by tBuCQN. Thus, only the fraction of analytes giving $\alpha<1.2$ (partial or no separation on tBuCQN CSP) was further considered (Supplementary Table S3). A Wilcoxon paired-rank test was performed for making the decision and the results are shown in Fig. 3 and Supplementary Table S4. This test confirms that cHexCQN and AdamCQN are not good choices. The former CSP does not provide significantly different distributions ( $P$-value 0.28845). The latter CSP shows a significant difference, but the rank sum is much better for tBuCQN (i.e. it gives better separation factors; in any case it was better than 1AdamCQN for the selected analytes). QN and N-Me-tBuCQN CSPs are not significantly different according to the Wilcoxon test. The rank sum of $\mathrm{QN}$ is less than the one of tBuCQN (i.e. the former CSP gives in the majority of cases lower separation factors within the selected analyte set with $\alpha<$ 1.2 on tBuCQN CSP). The N-Me tBuCQN CSP does also not show a significant difference in the Wilcoxon test but in this case the rank sum is higher than that of tBuCQN; it shows on average more cases with higher separation factors than on tBuCQN CSP. In case of DIPPCQN and TritCQN CSP, both exhibit significantly different separation factors and in both cases the rank sum is much lower for the tBuCQN CSP. According to statistics the performance is essentially par, yet the decision would be in favor of the TritCQN CSP (14 
positive ranks i.e. TritCQN gives larger separation factors vs only 2 negative ranks i.e. tBuCQN provides higher separation factors). For practical reasons, the final decision for the complementary CSP was DIPPCQN which is easier to synthesize (i.e. its synthesis needs less steps). Exemplary chromatograms of separations on DIPPCQN CSP are given in Fig. 4. For such analytes, hydroxycarboxylic acids, aryl carboxylic acids and small aliphatic acids without aromatic groups, the DIPPCQN CSPs turns out to be particularly suitable (Supplementary Table S5 and S6). In conclusion, by combining tBuCQN CSP and DIPPCQN CSP in a 2D-HPLC setup, a wider range of chiral compounds should be resolved into enantiomers and thus a better generic enantioselective HPLC system should be the result.

\subsubsection{Orthogonality of retention factors}

A mandatory requirement for $2 \mathrm{D}-\mathrm{HPLC}$ is sufficient orthogonality in the retention factors on the ${ }^{1} \mathrm{D}$ and ${ }^{2} \mathrm{D}$ separations. Thus, aside of extension of the enantioselectivity range by the second separation dimension, retention orthogonality needs to be realized to spread analyte enantiomer peaks in the 2D-separation space.

It can be assumed that CSPs with large distance in the score plot of a PCA analysis using retention factors as variables are dissimilar and thus more orthogonal. Consequently, a PCA study was performed. The model summary is given in Supplementary Table S7 and the biplot is depicted in Supplementary Fig. S1. By this model $93.3 \%$ of the variance can be explained by the model and the predictive power is reasonable $\left(Q^{2}(\mathrm{cum})=0.775\right.$ with two components). It can be seen in Supplementary Fig. S1 that the tBuCQN CSP and TritCQN CSP are located most distant from each other i.e. they are most dissimilar regarding their retention factors $k_{1}$. The DIPPCQN is nearly superimposed on the score of the TritCQN in the bi-plot. Consequently, it should be equally dissimilar in terms of retention factors. Hence, the DIPPCQN CSP is most likely a good choice as a second CSP in a chiral $\times$ chiral 2D-HPLC setup. 
To support the decision, Table 1 shows the correlation matrix of retention factors $k_{1}$ between the different CSPs. It can be seen that the correlations are all significant at the $\mathrm{P}=0.01$ significance level indicating poor orthogonality. However, there is clear trend: Pearson correlation coefficients with tBuCQN decline in the order cHexCQN $(r=0.943)>1$ AdamCQN $(0.920)>\operatorname{TritCQN}(0.833)>\mathrm{QN}(0.769)>\operatorname{DIPPCQN}(0.660)>N-M e-C Q N(0.645)$, i.e. orthogonality increases in this order. The two CSPS DIPPCQN and N-Me-CQN could be considered sufficiently complementary to be useful for 2D-HPLC. For broader enantioselectivity the DIPPCQN selection is preferred. These results support above discussed choice. The combination of tBuCQN CSP and DIPPCQN CSP appears to be the most promising selection, out of the tested CSPs, for a widely generic enantiomer separation system for chiral acids based on chiral×chiral 2D-HPLC.

\subsection{Implementation in enantioselective chiral $\times$ chiral 2D-HPLC}

\subsubsection{Proof of principle by test mixture}

For this proof of principle an amino acid test mixture derivatized with Sanger's reagent (yields 2,4-dinitrophenylated,DNP, amino acids) was selected. The labelling group per se is a structural variable that can be altered to achieve complementary retention and enantioselectivity profiles. For example, Boc and Fmoc amino acid derivatives used in peptide synthesis can be well separated by proposed 2D-HPLC as well, but will provide distinct 2D-plots [38, 39]. Likewise, 6-aminoquinolyl- $N$-hydroxysccinimidyl carbamate $(A Q C)$ reagent was proposed as suitable tagging strategy for enantiomer separation on quinine/quinidine carbamate CSPs as well [40]. DNP-amino acids have the advantage that both primary and secondary amino acids can be well resolved on quinine carbamate CSPS [38].

The DIPPCQN CSP (5 $\mu \mathrm{m}$ particles, $150 \times 4.0 \mathrm{~mm}$ ID) constituted the ${ }^{1} \mathrm{D}$-separation dimension. The tBuCQN CSP for ${ }^{2} \mathrm{D}$-separation was packed into a $33 \times 4.6 \mathrm{~mm}$ ID column to cope with the requirement of fast ${ }^{2} \mathrm{D}$ separations (60s or less). Discrete $(60 \mu \mathrm{L})$ fractions of 
$1^{\text {st }}$ dimension effluent were transferred to the $2^{\text {nd }}$ dimension using a 2-position 4-port dual valve as shown in Fig. 5 . In this 2D-setup, one loop is filled with effluent from column 1 while the other loop is emptied concurrently (i.e. same direction of loop filling and injection onto second column) into column 2 and analyzed in the second dimension. When the analysis in the second dimension is finished, the valve switches again and positions of upper and lower loop in the flow paths of dimension 1 and 2 are interchanged (modulation time 60s i.e. valve switching occurs every minute).

Stable-bond CSPS [36] were used, as ${ }^{2}$ D-separations required rather harsh conditions: Elevated temperature $\left(55^{\circ} \mathrm{C}\right)$ enabled fast $2^{\text {nd }}$ dimension separations of dinitrophenylated amino acids within 60 seconds. , It allowed a faster elution in the $1^{\text {st }}$ dimension, and thus the benefit was a shorter overall analysis time [41]. Strictly speaking, this setup constitutes a compromise between overall analysis time and (total) comprehensiveness, as $60 \mu \mathrm{L}$ were transferred each minute while actually operating the ${ }^{1} \mathrm{D}$ column at $100 \mu \mathrm{L} / \mathrm{min}$. A part of the collected fraction is therefore lost in each cut. The use of $3 \mathrm{~mm} I D{ }^{1} \mathrm{D}$-columns would totally compensate this drawback because at the same linear flow rate about $56.25 \mu \mathrm{L}{ }^{1} \mathrm{D}$-effluent would be switched each minute. Further optimizations (such as use of sub-2 $\mu$ m or superficially porous silica supports for fast ${ }^{2} \mathrm{D}$ separations, as well as optimization of column diameters and length in each dimension) are recommended for practical applications. For the proof of principle, we use here readily available columns ( $4 \mathrm{~mm}$ and $4.6 \mathrm{~mm}$ ID) and CSPs (5 $\mu \mathrm{m}$ fully porous particles).

The resultant 2D-separation is shown in Fig. 6a. It is evident that good enantiomer separations could be achieved. Unfortunately, however, orthogonality is not good enough. All the peaks are distributed along the diagonal line. In this configuration the 2D-HPLC setup is not very suitable for practical applications.

\subsubsection{Amino acid enantiomer separation by comprehensive 2D-HPLC with complementary CSPs and opposite configurations}


In the above test mixture of Fig. $6 a$, we may distinguish two independent structural variables in the analytes investigated, viz. amino acid residue (with differences in lipophilicity/hydrophilicity of side chains) and stereochemistry ( $D$ or $L$ configurations). Giddings suggested the term of sample dimensionality to describe "the number of independent variables that must be specified to identify the components of the sample" [42]. In the 2D-separation of Fig. 6a, we inappropriately deal with this twofold sample dimensionality, i.e. amino acids' residue and stereochemistry. The two CSPS (DIPPCQN and tBuCQN) are only slightly orthogonal because only one of the two sample dimensions is addressed by the selector structure variation, namely the amino acid residue. The different carbamate residue causes alteration in the retention factors and evidently, this CSP combination therefore does exhibit some orthogonality regarding the amino acid side chains. It shows, however, no orthogonality in terms of the stereochemistry because in both CSPS the same chiral selector backbone (i.e. quinine) is used which gives the same enantiomer elution order on both CSPs. To address also the second structural variable of the sample, the stereochemistry of the chiral selector in either one of the two separation dimensions must be "exchanged" to yield a chromatographic system exhibiting opposite elution orders. Practically, this can be conveniently realized by switching the stereochemistry of the chiral selector in the ${ }^{1} \mathrm{D}$-separation from quinine $(8 \mathrm{~S}, 9 \mathrm{R})$ to quinidine $(8 \mathrm{R}, 9 \mathrm{~S})$, i.e. substituting the DIPPCQN for the DIPPCQD CSP (Fig. 1). This slight modification significantly improves the orthogonality in the two separation dimensions, and significantly expands the coverage of the 2D-separation space. These improvements are clearly evident from a comparison of the 2DLC plots given in Fig. 6: While all the analytes elute close to the diagonal line with the DIPPCQN×tBuCQN 2D-HPLC system (poor orthogonality, see Fig. 6a), the DIPPCQD ×tBuCQN 2D-HPLC method allows efficient sampling of the 2D-space due to providing some complementarity in terms of amino acid side chain selectivity and perfect orthogonality in terms of stereorecognition and elution order, respectively (see Fig. 6b). In fact, the perfect complementarity of the tBuCQN CSP related to DIPPCQD in terms of stereoselectivity (i.e. reversed affinity and enantiomer elution order, respectively) avoids 
many peak overlaps. Consequently, in chiral×chiral 2D-HPLC separations with structurally related chiral selectors, it is worth considering the absolute configuration of the chiral selector(s) as an important experimental variable for maximizing chromatographic orthogonality.

\section{Conclusions}

The enantioselectivity of chiral selectors from the carbamoylated cinchonan family can be readily tuned by variation of the chemical nature and steric requirements of the carbamate functionality. Amongst the CSPs tested in this study, the selector incorporating the 2,6diisopropylphenyl carbamate functionality provided reasonable complementarity to the tertbutyl carbamate residue, and allowed for an expansion of the enantioselectivity spectrum compared to the tBuCQN CSP. As compared to the tBuCQN CSP, aryl carboxylic acids (profens), 2-hydroxyalkanoic acids, 2-hydroxyarylcarboxylic acids, and other compounds can be better resolved into enantiomers on CSPs based on O-9-(2,6diisopropylphenylcarbamoyl)-modified quinine and quinidine (DIPPCQN/QD). Considering these distinct differences in enantiomer separation characteristics, in this study an effort was made to combine these CSPs (tBuCQN and DIPPCQN) to generate a chiral $\times$ chiral 2D-HPLC system for a more convenient generic screening of chiral analytes. It was demonstrated that, owing to favourable complementarity between the chosen CSPs, analytes not resolved by one dimension could successfully be resolved in the other, yielding improved overall enantiomer separation success rates. From the 2D-HPLC perspective, however, it was found that altering also the absolute configurations in the employed chiral selectors provided significantly improved orthogonality due to elution order reversal, with the consequence of a more efficient sampling of the 2D-separation space and thus a reduced risk for peak overlaps. Besides other basic principles of 2D-HPLC, the potential of improving the outcome of chiral×chiral 2D-LC separation by appropriately using CSPs incorporating chiral selectors of opposing stereochemistry may be a convenient, yet underappreciated asset for method 
development. Unfortunately, the concept of enantiomeric CSPs is not available for the majority of the widely used CSPs which are commonly derived from the natural chiral pool. Most notably, the polysaccharide and macrocyclic antibiotic CSPs do not provide such an option. Cinchona alkaloid CSPs offer the selector molecule in pseudo-enantiomeric form with opposite absolute configurations in two of five stereogenic centers (those responsible for chiral recognition) which allows to realize this concept, although partly in imperfect manner. Fully synthetic selectors would have an advantage in this regard.

\section{Acknowledgements}

ML is grateful to Agilent Technologies for financial support through an Agilent Technologies Research Award.

\section{References}

[1] N.M. Maier, P. Franco, W. Lindner, Separation of enantiomers: needs, challenges, perspectives, J. Chromatogr. A 906 (2001) pp. 3-33.

[2] M. Lammerhofer, Chiral recognition by enantioselective liquid chromatography: mechanisms and modern chiral stationary phases, J. Chromatogr. A 1217 (2010) pp. 814-856.

[3] B. Chankvetadze, E. Yashima, Y. Okamoto, Dichloro-, dimethyl-, and chloromethylphenylcarbamate derivatives of cyclodextrins as chiral stationary phases for highperformance liquid chromatography, Chirality 8 (1996) pp. 402-407.

[4] T. Zhang, D. Nguyen, P. Franco, Reversed-phase screening strategies for liquid chromatography on polysaccharide-derived chiral stationary phases, J. Chromatogr. A 1217 (2010) pp. 1048-1055.

[5] T. Zhang, D. Nguyen, P. Franco, Enantiomer resolution screening strategy using multiple immobilised polysaccharide-based chiral stationary phases, J. Chromatogr. A 1191 (2008) pp. 214222.

[6] D.W. Armstrong, Y. Tang, S. Chen, Y. Zhou, C. Bagwill, J.-R. Chen, Macrocyclic Antibiotics as a New Class of Chiral Selectors for Liquid Chromatography, Anal. Chem. 66 (1994) pp. 1473-1484.

[7] A. Berthod, X. Chen, J.P. Kullman, D.W. Armstrong, F. Gasparrini, I. D'Acquarica, C. Villani, A. Carotti, Role of the Carbohydrate Moieties in Chiral Recognition on Teicoplanin-Based LC Stationary Phases Anal.Chem. 72 (2000) pp. 1767-1780.

[8] D.D. Schumacher, C.R. Mitchell, T.L. Xiao, R.V. Rozhkov, R.C. Larock, D.W. Armstrong, Cyclodextrin-based liquid chromatographic enantiomeric separation of chiral dihydrofurocoumarins, an emerging class of medicinal compounds, J. Chromatogr. A 1011 (2003) pp. 37-47.

[9] G. Toro, A. Peter, A. Gaucher, M. Wakselman, J.-P. Mazaleyrat, D.W. Armstrong, Highperformance liquid chromatographic separation of novel atropic $\alpha, \alpha$-disubstituted- $\beta$-amino acids, either on different $\beta$-cyclodextrin-bonded phases or as their 1-fluoro-2,4-dinitrophenyl-5-L-alanine amide derivatives, J. Chromatogr. A 846 (1999) pp. 83-91.

[10] M.H. Hyun, Liquid chromatographic enantioseparations on crown ether-based chiral stationary phases, J. Chromatogr. A 1467 (2016) pp. 19-32. 
[11] R. Berkecz, I. Ilisz, A. Misicka, D. Tymecka, F. Fulop, H.J. Choi, M.H. Hyun, A. Peter, HPLC enantioseparation of beta2-homoamino acids using crown ether-based chiral stationary phase, J. Sep. Sci. 32 (2009) pp. 981-987.

[12] M. Laemmerhofer, W. Lindner, Quinine and quinidine derivatives as chiral selectors. I. Brush type chiral stationary phases for high-performance liquid chromatography based on cinchonan carbamates and their application as chiral anion exchangers, J. Chromatogr. A 741 (1996) pp. 33-48. [13] A. Mandl, L. Nicoletti, M. Lammerhofer, W. Lindner, Quinine versus carbamoylated quininebased chiral anion exchangers. A comparison regarding enantioselectivity for $\mathrm{N}$-protected amino acids and other chiral acids, J. Chromatogr. A 858 (1999) pp. 1-11.

[14] W. Bicker, I. Chiorescu, V.B. Arion, M. Laemmerhofer, W. Lindner, Contributions to chromatographic chiral recognition of permethrinic acid stereoisomers by a quinine carbamate chiral selector: evidence from X-ray diffraction, DFT computations, 1 H NMR, and thermodynamic studies, Tetrahedron: Asymmetry 19 (2008) pp. 97-110.

[15] C.V. Hoffmann, R. Pell, M. Laemmerhofer, W. Lindner, Synergistic effects on enantioselectivity of zwitterionic chiral stationary phases for separations of chiral acids, bases, and amino acids by HPLC, Anal. Chem. 80 (2008) pp. 8780-8789.

[16] I. D'Acquarica, F. Gasparrini, B. Giannoli, E. Badaloni, B. Galletti, F. Giorgi, M.O. Tinti, A. Vigevani, Enantio- and chemo-selective HPLC separations by chiral-achiral tandem-columns approach: the combination of CHIROBIOTIC TAG and SCX columns for the analysis of propionyl carnitine and related impurities, J. Chromatogr. A 1061 (2004) pp. 167-173.

[17] R. Sardella, M. Lammerhofer, B. Natalini, W. Lindner, In-line coupling of a reversed-phase column to cope with limited chemoselectivity of a quinine carbamate-based anion-exchange type chiral stationary phase, J. Sep. Sci. 31 (2008) pp. 1702-1711.

[18] R.S. Hegade, M. De Beer, F. Lynen, Chiral stationary phase optimized selectivity liquid chromatography: A strategy for the separation of chiral isomers, J. Chromatogr. A 1515 (2017) pp. 109-117.

[19] K. Fried, I.W. Wainer, Column-switching techniques in the biomedical analysis of stereoisomeric drugs: why, how and when, J. Chromatogr. B: Biomed. Sci. Appl. 689 (1997) pp. 91-104.

[20] C. Lotter, E. Poehler, J.J. Heiland, L. Mauritz, D. Belder, Enantioselective reaction monitoring utilizing two-dimensional heart-cut liquid chromatography on an integrated microfluidic chip, Lab Chip 16 pp. 4648-4652.

[21] M. Penin-Ibanez, M.J. Santos-Delgado, L.M. Polo-Diez, Heart-cut achiral-chiral LC-LC method development using factorial design: application to the chiral separation of ketoprofen, Anal. Bioanal. Chem. 409 pp. 1135-1144.

[22] B. Kammerer, R. Kahlich, M. Ufer, S. Laufer, C.H. Gleiter, Achiral-chiral LC/LC-MS/MS coupling for determination of chiral discrimination effects in phenprocoumon metabolism, Anal. Biochem. 339 (2005) pp. 297-309.

[23] T. Welsch, C. Schmidtkunz, B. Mueller, F. Meier, M. Chlup, A. Koehne, M. Laemmerhofer, W. Lindner, A comprehensive chemoselective and enantioselective two-dimensional-HPLC set-up for fast enantiomer analysis of a multicomponent mixture of derivatized amino acids, Anal. Bioanal. Chem. 388 (2007) pp. 1717-1724.

[24] R. Koga, Y. Miyoshi, Y. Sato, M. Mita, R. Konno, W. Lindner, K. Hamase, Enantioselective determination of citrulline and ornithine in the urine of $d$-amino acid oxidase deficient mice using a two-dimensional high-performance liquid chromatographic system, J. Chromatogr. A 1467 (2016) pp. 312-317.

[25] L.M. Polo-Diez, M.J. Santos-Delgado, Y. Valencia-Cabrerizo, Y. Leon-Barrios, Simultaneous enantiomeric determinations of acid and ester imidazolinone herbicides in a soil sample by twodimensional direct chiral liquid chromatography, Talanta 144 (2015) pp. 375-381.

[26] Z. Deakova, Z. Durackova, D.W. Armstrong, J. Lehotay, Two-dimensional high performance liquid chromatography for determination of homocysteine, methionine and cysteine enantiomers in human serum, J. Chromatogr. A 1408 (2015) pp. 118-124. 
[27] F. Ianni, R. Sardella, A. Lisanti, A. Gioiello, B.T. Cenci Goga, W. Lindner, B. Natalini, Achiral-chiral two-dimensional chromatography of free amino acids in milk: A promising tool for detecting different levels of mastitis in cows, J. Pharm. Biomed. Anal. 116 (2015) pp. 40-46.

[28] E.L. Regalado, J.A. Schariter, C.J. Welch, Investigation of two-dimensional high performance liquid chromatography approaches for reversed phase resolution of warfarin and hydroxywarfarin isomers, J. Chromatogr. A 1363 (2014) pp. 200-206.

[29] S. Ma, N. Grinberg, N. Haddad, S. Rodriguez, C.A. Busacca, K. Fandrick, H. Lee, J.J. Song, N. Yee, D. Krishnamurthy, C.H. Senanayake, J. Wang, J. Trenck, S. Mendonsa, P.R. Claise, R.J. Gilman, T.H. Evers, Heart-Cutting Two-Dimensional Ultrahigh-Pressure Liquid Chromatography for Process Development: Asymmetric Reaction Monitoring, Org. Process Res. Dev. 17 (2013) pp. 806-810. [30] P. Dugo, M. Russo, M. Saro, C. Carnovale, I. Bonaccorsi, L. Mondello, Multidimensional liquid chromatography for the determination of chiral coumarins and furocoumarins in Citrus essential oils, J. Sep. Sci. 35 (2012) pp. 1828-1836.

[31] U. Woiwode, S. Neubauer, M. Kaupert, W. Lindner, M. Lämmerhofer, Trends in Enantioselective High Performance Liquid Chromatography, Supplement to LCGC Europe / LCGC North America 30 (2017) pp. 34-42.

[32] C.L. Barhate, E.L. Regalado, N.D. Contrella, J. Lee, J. Jo, A.A. Makarov, D.W. Armstrong, C.J. Welch, Ultrafast Chiral Chromatography as the Second Dimension in Two-Dimensional Liquid Chromatography Experiments, Anal. Chem. (Washington, DC, U. S.) 89 (2017) pp. 3545-3553. [33] D.R. Stoll, P.W. Carr, Two-Dimensional Liquid Chromatography: A State of the Art Tutorial, Anal. Chem. 89 (2017) pp. 519-531.

[34] A. Mandl, L. Nicoletti, M. Lämmerhofer, W. Lindner, Quinine- versus carbamoylated quininebased chiral anion exchangers: A comparison regarding enantioselectivity for N-protected amino acids and other chiral acids, J. Chromatogr. A 858 (1999) pp. 1-11.

[35] C. Rosini, C. Bertucci, D. Pini, P. Altemura, P. Salvadori, Cinchona alkaloids for preparing new, easily accessible chiral stationary phases.I. 11-(10,11-Dihydro-6'-methoxy-cinchonan-9-OL)tiopropylsilanized silica, Tetrahedron Lett. 26 (1985) pp. 3361-3364.

[36] A. Zimmermann, J. Horak, A. Sievers-Engler, C. Sanwald, W. Lindner, M. Kramer, M. Lammerhofer, Surface-crosslinked poly(3-mercaptopropyl)methylsiloxane-coatings on silica as new platform for low-bleed mass spectrometry-compatible functionalized stationary phases synthesized via thiol-ene click reaction, J. Chromatogr. A 1436 (2016) pp. 73-83.

[37] W. Bicker, M. Lammerhofer, W. Lindner, Direct high-performance liquid chromatographic method for enantioselective and diastereoselective determination of selected pyrethroic acids, J. Chromatogr. A 1035 (2004) pp. 37-46.

[38] M. Laemmerhofer, W. Lindner, Liquid chromatographic enantiomer separation and chiral recognition by cinchona alkaloid-derived enantioselective separation materials, Adv. Chromatogr. 46 (2008) pp. 1-107.

[39] G. Lajko, N. Grecso, G. Toth, F. Fulop, W. Lindner, A. Peter, I. Ilisz, A Comparative Study of Enantioseparations of N(alpha)-Fmoc Proteinogenic Amino Acids on Quinine-Based Zwitterionic and Anion Exchanger-Type Chiral Stationary Phases under Hydro-Organic Liquid and Subcritical Fluid Chromatographic Conditions, Molecules 21 (2016) pp.

[40] R. Hellinger, J. Horak, W. Lindner, Enantioseparation of 6-aminoquinolyl-N-hydroxysuccinimidyl carbamate tagged amino acids and other zwitterionic compounds on cinchona-based chiral stationary phases, Anal. Bioanal. Chem. 405 (2013) pp. 8105-8120.

[41] W.R. Oberleitner, N.M. Maier, W. Lindner, Enantioseparation of various amino acid derivatives on a quinine based chiral anion-exchange selector at variable temperature conditions. Influence of structural parameters of the analytes on the apparent retention and enantioseparation characteristics, J. Chromatogr. A 960 (2002) pp. 97-108.

[42] J.C. Giddings, Sample dimensionality: A predictor of order-disorder in component peak distribution in multidimensional separation, J.Chromatogr. A 703 (1995) pp. 3-15. 


\section{Figure captions:}

Fig. 1: Chiral stationary phases tested (note, selector structure drawn with stereochemistry of quinine, QN)

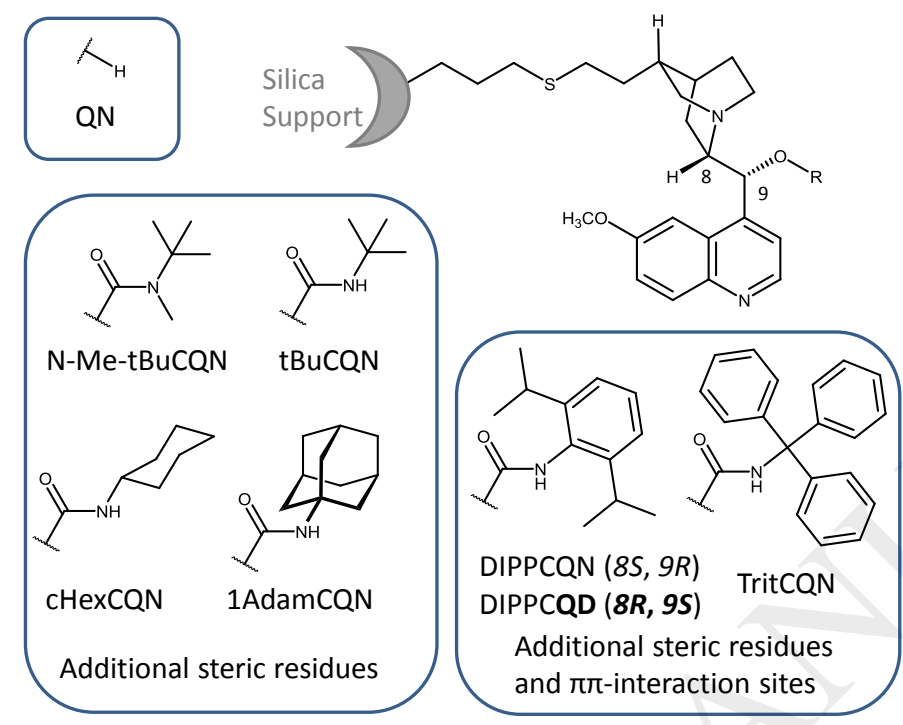

Fig. 1

Fig. 2: Complementarity of separation factors by PCA-X: Bi-Plot (a superposition of score plot, green dots, and loadings plot, black) of prinicipal components 1 and 2 . 


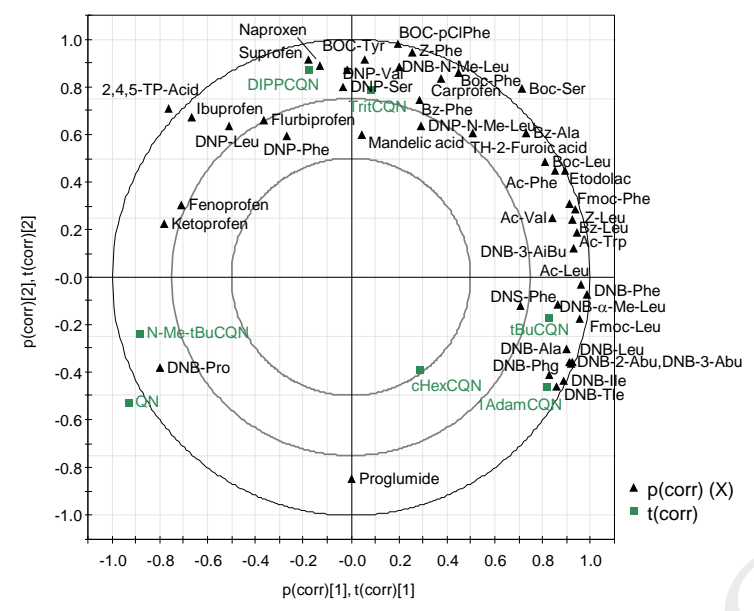

Fig. 2

Fig. 3: a) Distributions of separation factors for analytes giving $\alpha<1.2$ (partial or no separation) on tBuCQN CSP. b) Distributions of selectivity differences ( $\alpha$-value of tBuCQN subtracted from corresponding $\alpha$-value $\alpha_{x}$ of the same test compound on each CSP). Circles, $\alpha$-values (a) or $\alpha$-differences (b); black box, median value of data set in each column.
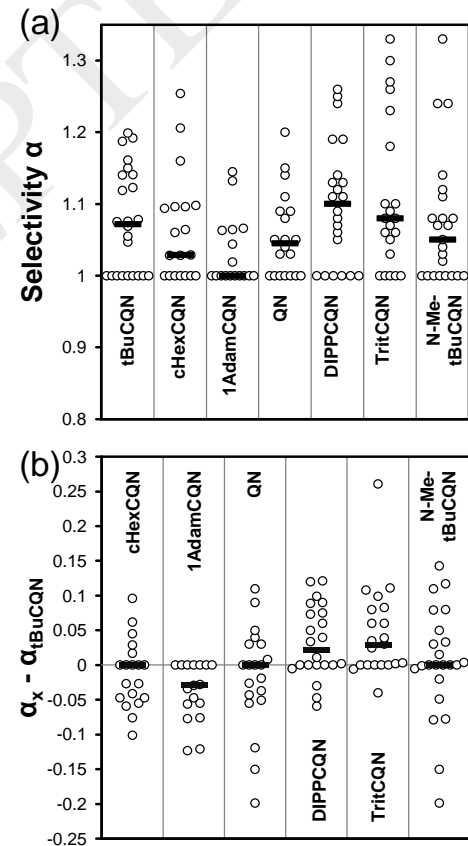

Fig. 3 
Fig. 4: Exemplary chromatograms of atrolactic acid (a) and tetrahydro-2-furoic acid (b) on DIPPCQN CSP. Conditions: Mobile phase, ACN-MeOH (50:50, v/v) containing 0.1\% $\mathrm{AcOH} ; \mathrm{T}, 15^{\circ} \mathrm{C}$; flow rate, $1 \mathrm{~mL} / \mathrm{min}$; detection, $230 \mathrm{~nm}$ and $\mathrm{ORD}$.
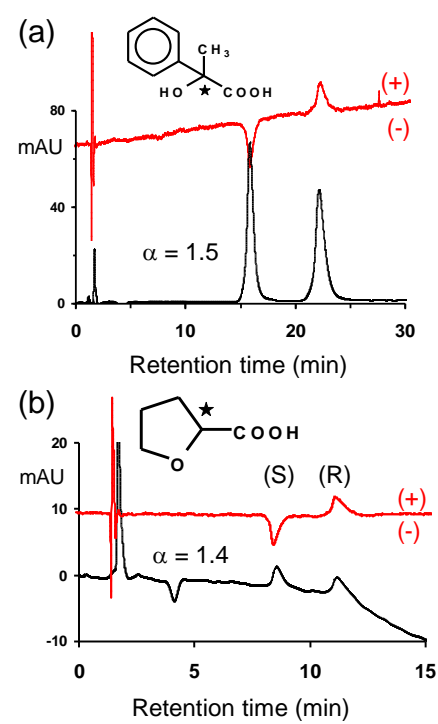

Fig. 4

Fig. 5: Dual 2-position/4-port valve (equipped with $60 \mu \mathrm{L}$ sample loops) for interfacing of $1^{\text {st }}$ and $2^{\text {nd }}$ chiral separation dimension.

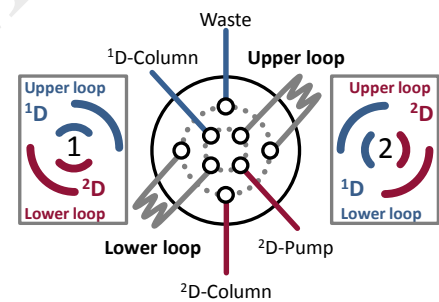

Fig. 5 
Fig. 6: Principle of 2D-HPLC exemplified by enantiomer separation of amino acid mixture derivatized with Sanger's reagent using a) complementary CSPs based on distinct carbamate residues (DIPPCQN in $1^{\text {st }}$ and tBuCQN in $2^{\text {nd }}$ dimension), and b) orthogonal CSPs with distinct carbamate residues and opposite absolute configuration in selector moiety i.e. quinine QN $(8 S, 9 R)$ vs quinidine QD $(8 R, 9 S)$ (DIPPCQD in $1^{\text {st }}$ and tBuCQN in $2^{\text {nd }}$ dimension). Capital letters indicate non-chiral or (S)-enantiomers and lower case letters $(R)$-enantiomers of amino acids denoted in one-letter code.

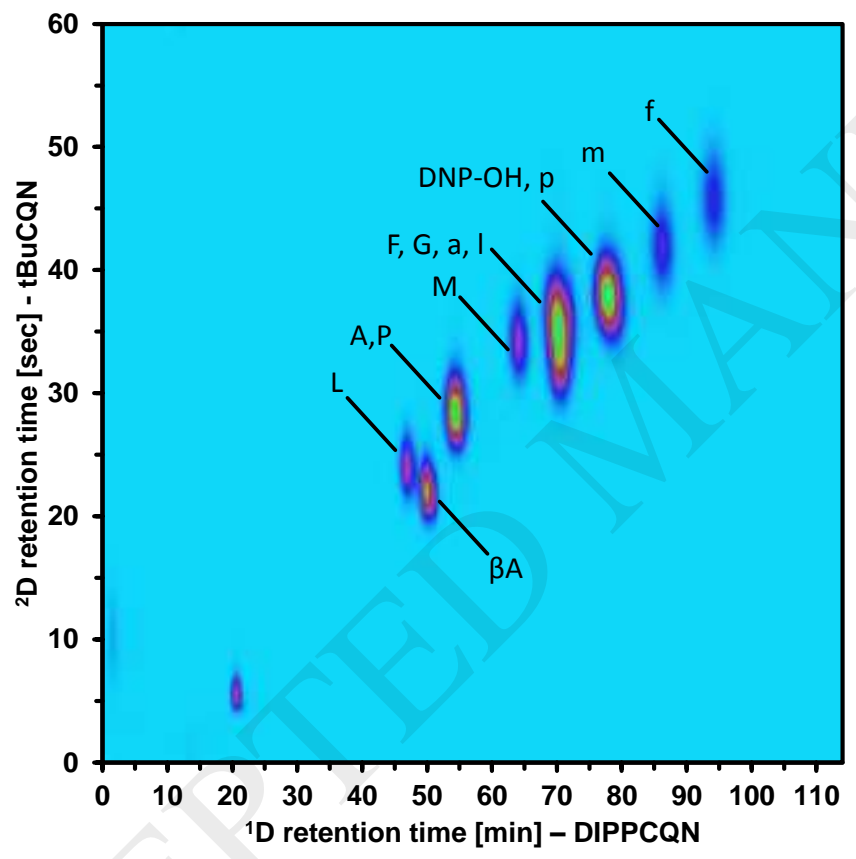

Fig. 6a 


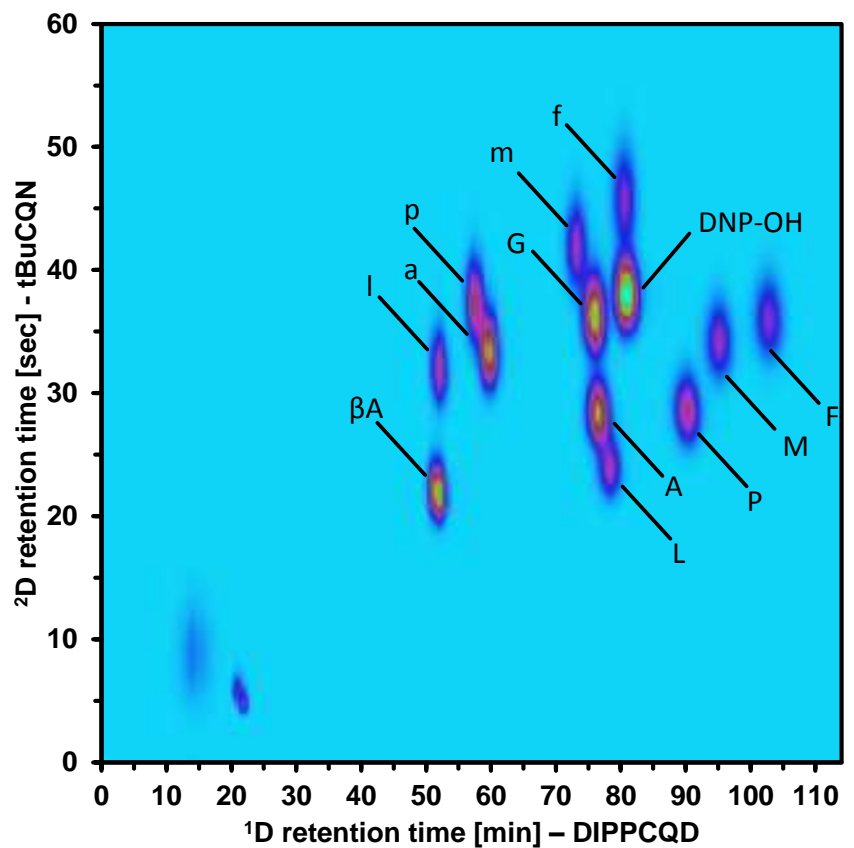

Fig. 6b ${ }^{1} \mathrm{D}$ retention time [min] - DIPPCQD 
Table 1. Correlation matrix of retention factors $k 1$ ( $r=$ Pearson correlation coefficient)

\section{Correlations}

\begin{tabular}{|c|c|c|c|c|c|c|c|c|}
\hline & & $\begin{array}{c}\text { tBuCQ } \\
N\end{array}$ & $\underset{N}{\operatorname{cHexCQ}}$ & $\begin{array}{c}\text { 1AdamC } \\
\text { QN }\end{array}$ & QN & $\begin{array}{c}\text { DIPPCQ } \\
\mathbf{N}\end{array}$ & $\begin{array}{c}\text { TritCQ } \\
\mathbf{N}\end{array}$ & $\begin{array}{c}N \text {-Me- } \\
\text { tBuCQ } \\
N\end{array}$ \\
\hline \multirow[t]{2}{*}{ tBuCQN } & $r$ & 1.000 & 0.943 & 0.920 & $\begin{array}{c}0.76 \\
9\end{array}$ & 0.660 & 0.833 & 0.645 \\
\hline & $\mathrm{N}$ & 57 & 49 & 43 & 52 & 57 & 57 & 57 \\
\hline \multirow[t]{2}{*}{ cHexCQN } & $r$ & 0.943 & 1.000 & 0.930 & $\begin{array}{c}0.76 \\
2\end{array}$ & 0.676 & 0.891 & 0.641 \\
\hline & $\mathrm{N}$ & 49 & 49 & 38 & 45 & 49 & 49 & 49 \\
\hline \multirow[t]{2}{*}{$\begin{array}{l}\text { 1AdamC } \\
\text { QN }\end{array}$} & $r$ & 0.920 & 0.930 & 1.000 & $\begin{array}{c}0.83 \\
6\end{array}$ & 0.791 & 0.912 & 0.769 \\
\hline & $\mathrm{N}$ & 43 & 38 & 44 & 39 & 44 & 44 & 44 \\
\hline \multirow[t]{2}{*}{ QN } & $r$ & 0.769 & 0.762 & 0.836 & $\begin{array}{c}1.00 \\
0\end{array}$ & 0.671 & 0.797 & 0.649 \\
\hline & $\mathrm{N}$ & 52 & 45 & 39 & 52 & 52 & 52 & 52 \\
\hline \multirow[t]{2}{*}{ DIPPCQN } & $r$ & 0.660 & 0.676 & 0.791 & $\begin{array}{c}0.67 \\
1\end{array}$ & 1.000 & 0.760 & 0.907 \\
\hline & $\mathrm{N}$ & 57 & 49 & 44 & 52 & 58 & 58 & 58 \\
\hline \multirow[t]{2}{*}{ TritCQN } & $r$ & 0.833 & 0.891 & 0.912 & $\begin{array}{c}0.79 \\
7\end{array}$ & 0.760 & 1.000 & 0.715 \\
\hline & $\mathrm{N}$ & 57 & 49 & 44 & 52 & 58 & 58 & 58 \\
\hline \multirow[t]{2}{*}{$\begin{array}{l}\text { N-Me- } \\
\text { tBuCQN }\end{array}$} & r & 0.645 & 0.641 & 0.769 & $\begin{array}{c}0.64 \\
9\end{array}$ & 0.907 & 0.715 & 1.000 \\
\hline & $\mathrm{N}$ & 57 & 49 & 44 & 52 & 58 & 58 & 58 \\
\hline
\end{tabular}

